\title{
用粪粒外部形态指标鉴定海南坡鹿的性别与年龄
}

\author{
李玉春 ${ }^{1,2, *}$, 蒙以航 ${ }^{2}$, 高海波 ${ }^{2}$, 孙苾芬 ${ }^{2}$, 张 海 $^{3}$, 林贤梅 ${ }^{3}$, 李善元 ${ }^{3}$ \\ (1. 山东大学 威海分校海洋学院, 山东 威海 264209; 2. 海南师范大学 生物系, 海南 海口 571158 ; \\ 3. 海南大田国家级自然保护区, 海南 东方 572600)
}

摘要: 利用粪便形态指标鉴定有蹄类动物的性别和年龄对研究不同性别年龄个体的生态学和性别分离具有重 要价值。利用 2006 年 3 月在海南大田国家级自然保护区采集的 145 头（次）已知性别和年龄组的野生海南坡鹿 (Cervus eldi hainanus) 粪便 4006 粒, 将坡鹿划分为成年雄鹿、成年雌鹿、亚成年雄鹿、亚成年雌鹿和幼鹿 5 个性 别年龄组, 使用逐步判别分析和聚类分析对粪粒的 4 项直接测度指标 (干重、体积、长轴长、短轴长) 以及 2 项 间接测度指标 (长短轴比和椭球形状指数) 进行分析。结果表明: 逐步判别分析对海南坡鹿粪粒的性别年龄组正 判率为成年雄鹿 $76.17 \%$ 、成年雌鹿 $42.22 \%$ 、亚成年雄鹿 $34.94 \%$ 、亚成年䧳鹿 $40.46 \%$ 、幼鹿 $79.34 \%$, 聚类分析 的判别率为成年雄鹿 $19.48 \%$ 、成年雌鹿 $20.02 \%$ 、亚成年雄鹿 $37.37 \%$ 、亚成年雌鹿 $42.82 \%$ 、幼鹿 $91.50 \%$ 。利用 粪粒形态判别海南坡鹿的性别年龄组最可靠的是幼鹿, 次之为成年雄鹿。利用粪粒形态判别海南坡鹿的性别年龄 组可以应用于以取样原理进行的群体水平研究, 但个体水平上的性别和年龄鉴定由于判别误差较大而难以应用。

\author{
关键词：海南坡鹿；粪粒形态；性别年龄鉴定；性别分离 \\ 中图分类号: Q959.83 文献标识码: A 文章编号: 0254-5853-(2008)02-000-06
}

\section{A Study on Determining Age and Sex of Hainan Eld's Deer by Use of Pellet Morphometry}

\author{
LI Yu-chun 1, 2, *, MENG Yi-hang ${ }^{2}$, GAO Hai-bo², SUN Rui-fen ${ }^{2}$, \\ ZHANG Hai ${ }^{3}$, LIN Xian-mei ${ }^{3}$, LI Shan-yuan ${ }^{3}$ \\ (1. Marine College, Shandong University at Weihai, Weihai 264209, China; 2. Department of Biology, Hainan Normal University, \\ Haikou 571158, China; 3. Hainan Datian National Nature Reserve, Dongfang 572600, China)
}

\begin{abstract}
Using morphometry of ungulate pellets to determine sex and age is the preferred method in field studies on ecological differences between two sexes, especially sexual segregation of ungulates. This study examined the morphometry of 4,006 pellets from $\leq 145$ Eld's Deer (Cervus eldi hainanus) collected in Hainan Datian National Nature Reserve in March 2006. The deer were divided into five sex-age categories as adult male, adult female, sub-adult male, sub-adult female, and calf. They were analyzed using four direct measurement indices (dry weight, length, width, and volume) and two indirect indices (length-to-width ratio, and ellipsoid-shape index) by use of step-wise discriminant and fuzzy cluster analyses. Discriminant analysis could correctly distinguish $76.17 \%$ of adult males, $42.22 \%$ of adult females, $34.94 \%$ of sub-adult males, $40.46 \%$ of sub-adult females, and $79.34 \%$ of calves. Fuzzy clustering analysis enabled to correctly distinguish $19.48 \%$ of adult males, $20.02 \%$ of adult females, $37.37 \%$ of sub-adult males, $42.82 \%$ of sub-adult females, and $91.50 \%$ of calves. Therefore, it is more credible to determine calves and secondarily adult males by use of pellet morphometry. We advise that pellet morphometry should be used for determining sex and age categories in surveys at the population level rather than at the individual level.
\end{abstract}

Key words: Cervus eldi hainanus; Pellet morphometry; Sex and age determination; Sexual segregation

利用野生动物的粪便研究动物的分布、生境利 用及密度估计等已在多种鹿科动物有所报道 (Neff, 1968; Ezcurra \& Gallina, 1981; Collins \& Urness,
1984; Rowland et al, 1984; Gallina et al, 1991; Leopold \& Krausman, 1991; Gallina, 1994; AlvarezCárdenas et al, 1999; Sánchez-Rojas \& Gallina,

收稿日期: 2008-01-06; 接受日期：2008-02-29

基金项目：国家自然科学基金资助项目（30360019,30660023）

“通讯作者(Corresponding author), 男, 教授, 主要从事动物生态学及其保护管理研究。E-mail: li_yuchun@hotmail.com 
2000a）。这些研究把各种动物作为一个物种对待, 并不涉及同种动物不同性别和年龄个体之间的生 态学差异。Clutton-Brock et al (1982)对鹿科动物䧳 雄个体生态学差异研究的确立, 使有蹄类动物不同 性别个体生境利用等生态学上的性别间差异研究 不断涌现 (Zhang \& Li, 2007), 以有蹄类性别分离 (sexual segregation)理论为典型代表, 提出了解释性 别分离现象的不同假说 (Ruckstuhl \& Neuhaus, 2000)。研究鹿科动物雌雄个体的生态学差异以及性 别分离, 需要鉴别不同性别和年龄个体的生境利用 技术。然而, 在野外条件下难以直接观察雌雄个体 的生境利用, 这就需要开发利用动物对生境的利用 痕迹或排泄物等来鉴别动物的性别与年龄的技术。 粪便是动物利用生境时的最常见排泄物, 因此利用 粪便鉴定排粪动物个体的性别与年龄, 对研究雌雄 个体的生境利用等生态学差异以及研究动物的性 别分离现象具有重应用价值(Sánchez-Rojas et al 2000b)。

利用鹿科动物粪便的外形特征和干重来鉴别排 粪个体性别和年龄的研究, 已见有以下数例报道:

Ezcurra \& Gallina (1981) 对白尾鹿 (Odocoileus virginianus)、 Bubenik (1982) 对马鹿 (Cervus elaphus)、MacCracken \& van Bellenberghe (1987)对 驼鹿(Alces alces)、Sánchez-Rojas et al (2004)对圈养 的黑尾鹿(Odocoileus hemionus)以及 Tumur \& Halik (2006) 对圈养的新疆马鹿 (Cervus elaphus yarkandensis) 的研究。这些研究表明, 不同性别和 年龄的个体其粪便外部形态和干重指标具有明显 差异, 特别是近年 Sanchez-Rojas et al (2000b)和 Tumur and Halik (2006)利用逐步判别分析进行研究 表明粪粒形态对鉴定鹿的性别年龄组具有较高的 正判率, 但这些研究使用了圈养个体鹿的粪便数 据, 且未包括亚成年雄鹿和䧳鹿。将这项技术应用 于野外研究, 还需要对野生非圈养鹿且包括亚成年 雄鹿和䧳鹿的结果。

海南坡鹿(Cervus eldi hainanus)是在我国仅分 布于海南岛的国家一级保护鹿科动物, 也是 CITES 附录 I 物种。我国对该物种的研究主要涉及行为、 食性与生境选择、种群动态与环境容纳量、驯养繁 殖以及保护利用、种群遗传多样性等方面 (Liu et al, 2007; Song, 1993; Song \& Li, 1991, 1992a, b;1995; Wang et al, 2005; Yuan et al, 1988, 1990, 1995; Zhang et al, 2007), 对不同性别年龄组的圈养泽鹿 $(C$. e. eldi)粪粒大小的性别及年龄组间的简单比较, 国 外有过报道(Khan \& Goyal, 1993), 但缺乏对该物种 野生种群以及用粪便外部形态鉴别性别与年龄的 研究。我们于 2006 年在海南大田国家级自然保护 区收集了海南坡鹿不同性别和年龄个体的粪便，对 利用粪便鉴别其性别和年龄进行了研究。

\section{1 研究方法}

\section{1 鹿粪采集}

本研究于 2006 年 3 月在海南大田国家级自然保 护区草地收集海南坡鹿粪便。采集粪便时由一名观 察人和一名采集人为一组, 观察人用望远镜观察在 草地采食的海南坡鹿个体, 发现排粪个体后以望远 镜将粪便定位, 并用手势等引导采集人准确无误地 找到粪便。每头排粪海南坡鹿个体采集粪粒约 30 粒, 放入塑料袋保存并将塑料袋开口放置使粪便自 然晾干后带回实验室处理。排粪海南坡鹿个体的性 别和年龄组分为成年雄鹿（以下亦简称成雄，下 同)、成年雌鹿（成䧳）、亚成年雄鹿（亚雄）、亚 成年雌鹿 (亚䧳)、幼鹿共 5 个性别年龄组。研究 期间共采集到 145 头（次）海南坡鹿的粪便, 其中 成雄 33、成䧳 27、亚雄 26、亚䧳 29、幼鹿 30 头 (次)。

\section{2 鹿粪处理与量度}

为了测定海南坡鹿粪粒干重, 随机选取 10 头 海南坡鹿的粪粒在恒温烘箱中以 $60^{\circ} \mathrm{C}$ 烘干并每隔 2 $\mathrm{h}$ 取出称重一次。经测定, 在 $60^{\circ} \mathrm{C}$ 恒温烘干 $6 \mathrm{~h}$ 后 海南坡鹿粪粒干重不再变化。因此, 我们采用 $60^{\circ} \mathrm{C}$ 恒温烘干 $6 \mathrm{~h}$ 后对海南坡鹿粪粒进行称重和测量。 对每粒海南坡鹿粪便用电子天秤称取干重 (精确到 $0.1 \mathrm{mg}$ ), 用游标卡尺测量其长轴长度 $(L)$ 和短轴长度 $(W)$, 最后将粪粒用细铁丝压入装有水的量筒中以 水的容积增加量测量粪粒体积 $(V)$ 。

\section{3 粪粒形状指标}

除了直接测度的粪粒干重 $(D)$ 、长轴长度 $(L)$ 、 短轴长度 $(W)$ 、体积 $(V) 4$ 个直接测度指标外, 还求 取了粪粒长宽比 $(G=L / W$, 长轴长度与短轴长度的 比值)、椭球形状指数 $(S)$ 二个形状指标作为粪粒外 形指标进行分析。本研究采用以下公式计算椭球形 状指数:

$$
S=\frac{V}{V^{\prime}}=\frac{6 V}{L W^{2} \pi}
$$

其中 $V$ 为粪粒的实测体积, $V^{\prime}$ 为粪粒的理想椭球体 
积, 即按椭球体积公式以长轴长度 $(L)$ 和短轴长度 $(W)$ 计算出的理想椭球体积。

\section{4 性别年龄组划分}

将海南坡鹿按照不同的性别年龄组划分进行 粪粒性别年龄鉴定。(1) 第一分组法: 划分为 5 个 年龄组, 即按照坡鹿大小和外形划分为成雄、成雌、 亚雄、亚雌、幼鹿。这是根据海南坡鹿外部形态能 够划分的最详细和野外研究最常用的性别年龄组 划分, 也是能够最详细和最具体评价粪粒对坡鹿不 同性别年龄组判别率的分组方法; (2) 第二分组法: 划分为 3 个年龄组, 即成雄、成雌和幼鹿。该分组 法不包括亚雄和亚雌个体, 因此这种方法在应用于 野外研究时有所欠缺。我们进行这种分组法的目 的, 是为了与已有报道(Sanchez-Rojas et al, 2004; Tumur \& Halik, 2006)进行比较。

\section{5 数据处理}

对不同性别年龄组坡鹿的粪粒各指标进行单 因素方差分析(ANOVA), 以确定其组间差异显著 性。如果不同性别年龄海南坡鹿的粪粒具有显著差 异, 进而进行判别分析和聚类分析。使用逐步判别 分析对所有已知性别年龄的海南坡鹿粪粒进行分 类, 求出判别函数并计算出对不同性别年龄组坡鹿 的判别率 (正判率)。逐步判别分析采用 Wilks' Lambda 方法, 变量引入和剔除分别采用 $\alpha=0.05$ 和 $\alpha=0.10$ 作为显著性概率标准。采用聚类分析对粪粒 样品进行与二种性别年龄组分组组数相同的聚类, 其每类的粪粒数设定为该性别年龄组的样本粪粒 数, 根据不同性别年龄组的实际粪粒数 $(F)$ 与被聚类 为对应组的粪粒数 $\left(F^{\prime}\right)$ 求取正判率 $\left(F^{\prime} / F \times 100 \%\right)$ 。以 上计算均使用 SPSS 11.0 软件完成。

\section{2 结 果}

海南坡鹿粪粒 $(n=4006)$ 的各项外形指标为：干 重 $(0.20 \pm 0.08) \mathrm{g}$ 、长轴长 $(11.50 \pm 1.92) \mathrm{mm}$ 、短轴长 $(8.81 \pm 1.99) \mathrm{mm}$ 、体积 $(454.90 \pm 203.99) \mathrm{mm}^{3}$ 、长短轴 比 $1.36 \pm 0.32$ 、椭球形状指数 $0.93 \pm 0.16$ 。粪粒各指 标的单因素方差分析表明, 不同性别年龄组粪粒的 各指标均存在显著性差异 (干重: $F_{(4,4001)}=2478.400$, $P<0.001$; 长轴: $F(4,4001)=225.484, P<0.001$; 短轴: $F(4,4001)=1824.337, P<0.001$; 体积: $F(4,4001)$ $=1846.110, P<0.001$; 长短轴比: $F(4,4001)=482.641$, $P<0.001$; 形状指数: $F(4,4001)=18.554, P<0.001$ 。

\section{1 粪粒的逐步判别分析结果}

2.1.1 粪粒各指标对判别函数的贡献率 二种性 别年龄组划分方法的第一判别函数贡献率分别为 $86.5 \%$ 和 $92.0 \%$, 均超过 $80 \%$ 。因此, 我们仅选取 第一判别函数作为识别粪粒性别年龄组的判别函 数式, 分别为:

第一分组法 $(G M 1): Y_{I}=23.59 D+0.18 L+$ $0.28 W+2.34 S-1.84 G-0.01 V-6.74$

第二分组法 $(G M 2): Y_{2}=21.09 D+0.60 L+$ $0.08 \mathrm{~W}+3.24 S-4.88 G-0.01 V-5.89$

根据第一判别函数使用各性别年龄组的各粪 粒指标平均值计算出不同性别年龄组的判别区间 如下: 第一分组法 $(G M 1)$ : 成雄 $[+\propto,-1.56]$ 、成雌 [-1.57, -1.99]、亚雄[-2.00, -2.47]、亚雌[-2.48, -3.29]、 幼鹿 $[-3.30,-\infty]$; 第二分组法 $(G M 1)$ ：成雄 $(+\infty$, -1.16]、成雌[-1.17, -2.85]、幼鹿 $[-2.86,-\propto]$ 。

在第一分组法中, 粪粒各指标在判别函数式中 的得分（绝对值）从大到小依次是干重 $(D, 1.04)$ 、 体积 $(V,-0.60)$ 、长宽比 $(G,-0.48)$ 、椭球形状指数 $(S$, $0.37)$ 、短轴 $(W, 0.33)$ 、长轴 $(L, 0.31)$, 干重是判别粪 粒所属性别年龄组的最主要影响因子, 表明不同性 别年龄组间粪粒的变化最明显的是粪粒干重。实测 体积排在第 2 位, 表明与干重的高度一致性。其次 是表示粪粒形状的指标长宽比和椭球形状指数, 最 后才是短轴和长轴。所以, 仅以粪粒短轴和长轴大 小判断粪粒所属组别误差最大。由此可见, 不同性 别年龄组粪粒的判别要素主要是表示粪粒大小的 干重和体积, 其次是形状指标, 粪粒的长轴和短轴 最差。

在第二分组法中, 粪粒各指标在判别函数式中 的得分 (绝对值) 从大到小依次是长宽比 $(G,-1.26)$ 、 长轴 $(L, 1.06)$ 、干重 $(D, 0.87)$ 、体积 $(V,-0.61)$ 、椭球 形状指数 $(S, 0.51)$ 、短轴 $(W, 0.09)$, 椭球形状指数长 宽比成为判别粪粒所属性别年龄组的最主要影响 因子, 长轴次之。因此, 在剔除了亚雄和亚雌这两 个中间性年龄组后, 海南坡鹿的粪粒判别函数发生 变化, 说明粪粒干重和体积在亚雄和亚雌两个性别 年龄组之间以及与其他组之间具有较大的重叠性。

2.1.2 逐步判别分析对粪粒的判别率 第一分组 法各性别年龄组坡鹿粪粒的正判率为: 成雄 $76.17 \%$ 、成雌 $42.22 \%$ 、亚雄 $34.94 \%$ 、亚䧳 $40.46 \%$ 、 幼鹿 $79.34 \%$ (表 1), 成雄和幼鹿的正判率最高, 均超过 $75.0 \%$ 。从误判率的分配可知, 成雄主要被 误判为成雌 $(16.44 \%)$, 少数被误判为亚雄 $(7.27 \%)$, 
没有粪粒被误判为亚䧳和幼鹿; 成雌最多地被误判 为亚雄 $(26.67 \%)$, 但被误判为成雄 $(16.34 \%)$ 和亚䧳 $(16.77 \%)$ 的也较多, 没有粪粒被误判为亚雌和幼鹿; 亚雄最多地被误判为成雌 $(32.32 \%)$, 也有许多被误 判为成雄 $(16.99 \%)$, 亚䧳 $(15.61 \%)$ 的也较多, 仅有 1 粒 $(0.14 \%)$ 被误判为幼鹿; 亚雌被误判为幼鹿的为最 多 $(30.28 \%)$, 较多地被误判为亚雄 $(18.17 \%)$ 和成䧳 $(10.70 \%)$, 仅有 3 粒 $(0.39 \%)$ 被误判为成雄; 幼鹿主 要被误判为亚雌 $(16.88 \%)$, 很少被误判为成䧳 $(1.06 \%)$ 和亚雄 $(2.72 \%)$, 没有被误判为成雄的粪粒。

第二分组法各性别年龄组坡鹿粪粒的正判率 为：成雄 $77.29 \%$ 、成雌 $81.96 \%$ 、幼鹿 $93.98 \%$ （表 2), 幼鹿具有最高的正判率。与第一分组法相比,
其 3 个性别年龄组的正判率均有提高, 特别是成䧳 的正判率大幅度提高。因此，在缺乏亚雄和亚䧳个 体的条件下，高的正判率并不能说明对全部性别年 龄组的正判率较高。

由以上结果可以看出, 用粪粒形态指标判别不 同性别年龄组的坡鹿，成雄和幼鹿具有最高的正判 率，而其他性别年龄组（成䧳、亚雄、亚雌）的正 判率则相对较低。

\section{2 聚类分析的判别率}

运用快速聚类分析对两种性别年龄组分类的 聚类结果表明（表 3 )，幼鹿的正判率较高（均大于 $91 \%$ ) 外, 其他 4 个性别年龄组的判别率均较低 (均 小于 $70 \%$ )。其中性别年龄类别最多的第一分组法

表 1 不同性别年龄组海南坡鹿粪粒的正判率与误判率 (\%) 分布（第一分组法）

Tab. 1 Distinguished in all sex-age groups by using discriminant function analysis (5 sex-age classes)

\begin{tabular}{lcccccc}
\hline $\begin{array}{c}\text { 性别年龄组 } \\
\text { Age \& Sex }\end{array}$ & $\begin{array}{c}\text { 粪粒数 } \\
\text { Pellet No. }\end{array}$ & $\begin{array}{c}\text { 成雄 } \\
\text { Adult male }\end{array}$ & $\begin{array}{c}\text { 成雌 } \\
\text { Adult female }\end{array}$ & $\begin{array}{c}\text { 亚雄 } \\
\text { Sub-adult male }\end{array}$ & $\begin{array}{c}\text { 亚雌 } \\
\text { Sub-adult male }\end{array}$ & $\begin{array}{c}\text { 幼鹿 } \\
\text { Calf }\end{array}$ \\
\hline 成雄 Adult male & 894 & $\mathbf{7 6 . 1 7}(681)$ & $16.44(147)$ & $7.27(65)$ & $0.11(1)$ & $0 \%(0)$ \\
成雌 Adult female & 765 & $16.34(125)$ & $\mathbf{4 2 . 2 2 ( 3 2 3 )}$ & $26.67(204)$ & $14.77(113)$ & $0 \%(0)$ \\
亚雄 Sub-adult male & 724 & $16.99(123)$ & $32.32(234)$ & $\mathbf{3 4 . 9 4 ( 2 5 3 )}$ & $15.61(113)$ & $0.14 \%(1)$ \\
亚雌 Sub-adult female & 776 & $0.39(3)$ & $10.70(83)$ & $18.17(141)$ & $\mathbf{4 0 . 4 6 ( 3 1 4 )}$ & $30.28 \%(235)$ \\
幼鹿 Calf & 847 & $0 \%(0)$ & $1.06(9)$ & $2.72(23)$ & $16.88(143)$ & $\mathbf{7 9 . 3 4 \% ( 6 7 2 )}$ \\
\hline
\end{tabular}

对角线上的粗体字百分率 $(\%)$ 为各性别年龄组正判率; 括号中的数字为粪粒数。

The bold percentages in diagonal are correctly discriminated rate of each sex-age groups; number in (\%) are pellets number.

表 2 不同性别年龄组海南坡鹿粪粒的正判率与误判率 (\%) 分布 (第二分组法)

Tab. 2. Distinguished in all sex-age groups by using discriminant function analysis (3 sex-age classes)

\begin{tabular}{lcccc}
\hline \multicolumn{1}{c}{ 性别年龄组 Age \& Sex } & 粪粒数 Pellet No. & 成雄 Adult male & 成雌 Adult female & 幼鹿 Calf \\
\hline 成雄 Adult male & 1618 & $\mathbf{7 7 . 2 9}(691)$ & $22.71(203)$ & $0(0)$ \\
成雌 Adult female & 1541 & $17.91(137)$ & $\mathbf{8 1 . 9 6 ( 6 2 7 )}$ & $0.13(1)$ \\
幼鹿 Calf & 847 & $0(0)$ & $6.02(51)$ & $\mathbf{9 3 . 9 8 ( 7 9 6 )}$ \\
\hline
\end{tabular}

对角线上的粗体字百分率 $(\%)$ 为各性别年龄组正判率; 括号中的数字为粪粒数。

The bold percentages in diagonal are correctly discriminated rate of each sex-age groups; number in () are pellet number.

表 3 不同性别年龄组坡鹿的聚类分析正判率

Tab. 3 Correctly discriminated percentages of 5 sex-age classes by Cluster analysis

\begin{tabular}{lccc}
\hline \multirow{2}{*}{ 年龄性别 Age \& Sex } & 粪粒数 & \multicolumn{2}{c}{ 正判率 Correctly discriminated(\%) } \\
\cline { 3 - 4 } & Pellet No. & GM1 & GM2 \\
\hline 成雄 Adult male & 894 & 19.48 & 61.07 \\
成雌 Adult female & 765 & 20.02 & 69.54 \\
亚雄 Sub-adult male & 724 & 37.37 & $*$ \\
亚雌 Sub-adult female & 776 & 42.82 & $*$ \\
幼鹿 Calf & 847 & 91.50 & 95.16 \\
\hline
\end{tabular}

*亚雄和亚雌没有被包括在分析中 (见原文)。

*Sub-adult male and Sub-adult female are excluded from analysis (see context). 
正判率最低。所以, 利用粪粒形态对坡鹿的年龄进 行鉴别, 幼鹿是最准确判定的年龄组, 而其他性别 年龄组则鉴别率均较低。

\section{3 讨 论}

\section{1 海南坡鹿粪粒形状特征}

从海南坡鹿粪粒的外部形态特征可以看出, 成 年雄鹿粪粒的各个指标都大于其他性别年龄组的 个体, 其粪粒形状最接近于球形 (长短轴的比例最 小）, 而幼鹿的粪粒外形细长而最趋于长椭球形。 其他 3 个性别年龄组的粪粒外形无论大小还是形状 介于成年雄鹿和幼鹿之间。因此, 可以说海南坡鹿 粪粒的形态结构取决于个体的体形大小, 即体形越 大粪粒则越大且越趋于圆球型, 体形越小则粪粒越 小且形状更趋于长椭球体。粪粒的这种外形特征决 定了利用粪粒外形指标鉴别排粪个体性别年龄组 的基础。

\section{2 影响粪粒鉴别性别年龄的因素}

Sanchez-Rojas et al (2004)利用粪粒外部性太对 黑尾鹿成年雄鹿、成年雌鹿和幼鹿三个性别年年龄 组的判别率分别为 $100 \% 、 91.66 \% 、 75 \%$, Tumur \& Halik (2006)对新疆马鹿进行了相同的研究, 得出对 成年雄鹿、成年雌鹿和幼鹿的判别率分别为 $100 \%$ 、 $89.33 \%$ 和 $75 \%$ 。 以上的研究使用的性别年龄组没有 包括亚成年雄鹿和亚成年雌鹿, 与本研究的第二分 组法相同。本研究的结果中成年雄鹿、成年雌鹿和 幼鹿的正判率分别为 $77.29 \% 、 81.96 \% 、 93.98 \%$, 与以上研究相比正判率在成年雄鹿较大程度偏低、 成年雌鹿稍低、而幼鹿则明显较大偏高。成年鹿的 差异可能是由不同鹿种身体大小及其雌雄间差异 (即性别体二形现象) 不同引起的, 黑尾鹿和马鹿 的体形要比海南坡鹿大得多, 这两种鹿的成年雄鹿 正判率均达到 $100 \%$, 而海南坡鹿仅为明显变低 (77.29\%)。本研究中幼鹿的判别率高于黑尾鹿和马 鹿, 其原因可能是在我们收集粪便的季节时幼鹿出 生后约 4 个月左右, Sanchez-Rojas et al (2004)研究 的黑尾鹿幼鹿和 Tumur \& Halik (2006)研究的马鹿 幼鹿在粪便收集季节可能幼鹿月龄大于本研究的 坡鹿幼鹿。另外, 还有一个重要区别, 即以上两个 研究均为人工圈养个体, 而本研究的坡鹿为野生个 体, 人工圈养个体的食物种类的单调性是否造成以 上差异亦不可排除。

另外, 食物种类的多少与含水量对粪粒的大小
和现状会有一定的影响, 因此不同季节粪粒的大小 和形状会有变化。这种变化的大小可能造成不同季 节的粪粒要采用不同的判别标准。海南岛明显区分 为雨季和旱季, 本研究为旱季的采样结果, 雨季海 南坡鹿的粪粒形状与大小以及各个性别年龄组的 正判率还需要进一步研究。

\section{3 用粪粒鉴别动物性别年龄的应用局限性和可 行性}

现有研究的性别年龄组分组方法中排除了亚 成年雄鹿和亚成年雌鹿个体, 所以在野外研究中实 际应用价值明显欠缺。本研究的第一分组法 (即划 分为成雄、成雌、亚雄、亚雌、幼鹿）具有最好的 应用价值, 但性别年龄组的正判率明显下降。这是 由于包含了亚成年雄鹿和亚成年雌鹿个体后, 不同 性别年龄组之间的粪粒指标重叠度增加, 导致正判 率下降。因此, 利用粪粒形态判别坡鹿的性别年龄 组具有较大的误差, 难以应用于对性别和年龄鉴别 要求准确的应用研究以及在动物个体水平上性别 年龄鉴别。但是, 对于依据取样原理进行的群体水 平研究 (如不同性别年龄组的生境利用)，利用粪 粒形态判别坡鹿的性别年龄组具有一定的应用价 值。

\section{4 粪粒形状指标和数据分析方法的选择}

Sanchez-Rojas et al (2004)和 Tumur \& Halik (2006) 利用圆柱体积公式求取粪粒体积作为粪粒 外形指标参加逐步判别分析, 我们的分析结果说明 坡鹿粪粒更接近于椭球。我们采用直接测量粪粒体 积的方法, 在以上两个研究的基础上增加了直接测 度指标粪粒体积和间接测度指标椭球形状指数（椭 球指数), 且这两个指标对判别函数具有较高效率 得分, 说明这两个指标在利用粪粒形态学指标判别 坡鹿性别年龄组上具有重要意义。聚类分析结果对 验证已知性别年龄组个体的坡鹿粪粒具有重要作 用, 但不便于应用于未知粪粒的判别。判别分析给 出判别函数, 容易直接适用于未知粪粒的判别, 适 于作为野外坡鹿粪粒的性别年龄组判别。

\section{5 粪粒法最适合鉴别幼鹿}

本研究揭示, 利用粪粒形态判别幼鹿的正判率 明显高于其他性别年龄组, 且幼鹿的粪粒明显小于 其他性别年龄组, 在外形上具有小而细长的显著性 特征, 在野外研究中最为易于判别, 对研究海南坡 鹿幼鹿的生境利用等具有重要应用价值。 


\section{参考文献:}

Alvarez-Cárdenas S, Gallina S, Gallina-Tessaro P, Dominguez-Cadena R. 1999. Habitat availability for the mule deer (Cervidae) population in a relictual oak-pine forest in Baja California Sur, Mexico [J]. Tropical Zoology, 12(1): 67-78.

Bubenik RA. 1982. Ecology and Management[A]. In: Thomas JW, Toweill DE. Physiology [M]. Elk of North America. Harrisburg, PA: Stackpole Books, 125-179.

Clutton-Brock TH, Guinness FE, Albon SD. 1982. Red Deer: Behaviour and Ecology of Two Sexes [M]. Chicago: Chicago University Press.

Collins WB, Urness PJ. 1984. The pellet-group census technique as indicator of relative habitat use: Response to Leopold et al [J]. Wildlife Society Bulletin, 12(3): 325-326.

Ezcurra E, Gallina S. 1981. Biology and population dynamics of white-tailed deer in northwestern Mexico [M]. In: Folliott PF, Gallina S. Deer biology, habitat requirements, and management in western North America[M]. Xalapa, Mexico: Instituto de Ecologia, 78-108

Gallina S, Galina-Tessaro P, Alvarez-Cardenas S. 1991. Mule deer density and pattern distribution in the pine-oak forest at the Sierra de la Laguna in Baja California Sur, México [J]. Ethology Ecology \& Evolution, 3: 27-33

Gallina S. 1994. Dinámica Poblacional del Venado. cola blanca en la reserva de la biosfera La. Michilia, Durango, México [A]. In: Vaughan C, Rodriguez MA. Ecologia y manejo del venado cola blanca en Me xico y Costa Rica[M]. EUNA: Costa Rica, 207-234.

Khan I, Goyal SP. 1993. Fecal pellet measurements of Manipur brow-antlered deer with respect to sex and age classes [J]. Zoo Biology, 12(6): 561-565.

Leopold BD, Krausman PR. 1991. Factors influencing desert mule deer distribution and productivity in southwestern Texas [J]. The Southwestern Naturalist, 36(1): 67-74.

Liu ZT, Ding JH, Song YL, Zeng ZG, Zhang Q. 2007. Wallowing behavior of Hainan Eld's deer Cervus eldi hainanus male during the rut and its function in reproduction [J]. Acta Zoologica Sinica, 53(3): 417-424. [刘志涛, 丁建华，宋延龄，曾治高，张 琼. 2007. 雄性海南坡鹿 发情期泥浴、沙浴行为及其在繁殖中的作用. 动物学报, 53(3): 417-424.]

MacCracken JG, Van Ballenbergher V. 1987. Age and sex-related differences in faecal pellet dimension of moose [J]. Journal of Wildlife Management, 51(2): 360-364.

Neff DJ. 1968. The pellet-group count technique for big game trend, census, and distribution: A review [J]. Journal of Wildlife Management, 32(3): 597-614.

Rowland MM, White G C, Karlen E M. 1984. Use pellet-group plots to measure trends in deer and elk populations [J]. Wildlife Society Bulletin, 12(2): 147-155.

Ruckstuhl KE, Neuhaus P. 2000. Sexual segregation in ungulates: A new approach [J]. Behaviour, 137: 361-377.

Sánchez-Rojas G, Gallina S, Equihua M. 2004. Pellet morphometry as a tool to distinguish age and sex in the mule deer [J]. Zoo Biology, 23(2): 139-146.

Sánchez-Rojas G, Gallina S. 2000a. Mule deer (Odocoileus hemionus) density in a landscape element of the Chihuahuan desert, Mexico [J]. Journal of Arid Environments, 44(3): 357-368.

Sánchez-Rojas G, Gallina S. 2000b. Factors affecting habitat use by mule deer (Odocoileus hemionus) in the central part of the Chihuahuan Desert, Mexico: An assessment with univariate and multivariate methods [J]. Ethology Ecology \& Evolution, 12: 405-417.

Song YL. 1993. Diurnal activity rhythms of Eld's deer on Hainan Island, China [A]. In: Ohtaish N, Sheng HL. Deer of China: Biology and Management[C]. Elsevier Science Publishers BV, 214-219.

Song YL, Li SY. 1991. The Selection for the Bedding Rest Places by the Fawn of Hainan Eld's Deer (Cervus eldi hainanus) [J]. Acta Theriologica Sinica, 11(3): 161-164. [宋延龄, 李善元. 1991. 海南 坡鹿仔鹿(Cervus eldi hainanus)对卧息地的选择. 兽类学报, 11(3): 161-164.]

Song YL, Li SY. 1992a. A food habits study on Hainan Eld's Deer in Hainan Island, China [J]. Acta Theriologica Sinica, 12(4): 148-154. [宋延龄, 李善元. 1992a. 海南坡鹿的食性研究. 兽类学报, 12(4): 148-154.]

Song YL, Li SY. 1992b. On Population dynamics of Hainan Eld's Deer (Cervus eldi hainanus) in Datian Nature Reserve, Hainan [J]. Acta Zoologica Sinica, 38(2): 165-171. [宋延龄, 李善元. 1992b. 海南大 田自然保护区海南坡鹿种群动态研究. 动物学报, 38(2): 165-171.]

Song YL, Li SY. 1995. Estimating carrying capacity of Hainan Datian National Nature Reserve for Eld's Deer [J]. Acta Zoologica Sinica, 41(3): 275-281. [宋延龄, 李善元. 1995. 海南大田国家级自然保护 区海南坡鹿容纳量的研究. 动物学报, 41(3): 275-281.]

Tumur A, Halik M. 2006. Using pellet morphometry study population age structure and sex of the red deer (Cervus elaphus yarkandensis) [J]. Life Science Research, 10(1): 61-66. [艾尼瓦尔·吐米尔，马合木提 哈.力克. 2006. 用粪便形态特征初步研究新疆塔里木马鹿种群年 龄和性别. 生命科学研究, 10(1): 61-66.]

Wang LJ, Hong ML, Chen XJ. 2005. Diurnal time budget and activity rhythm of Hainan Eld's Deer Fawns under different breeding conditions during spring [J]. Acta Theriologica Sinica, 25(1): 9-13.[ 王力军, 洪美玲, 陈兴军. 2005. 不同饲养条件下海南坡鹿 幼体春季昼间行为时间分配及活动节律. 兽类学报, 25(1): 9-13.]

Yuan XC, Liu XM, Wang J, Fu G, Li SY. 1990. Feeding habits of Hainan Thamin deer [J]. Journal of Northeast Forestry University, 8(1): 66-71. [袁喜才, 刘晓明, 王 骏, 符国瑗, 李善元. 1990. 海南坡 鹿食性的研究. 东北林业大学学报, 8(1): 66-71.]

Yuan XC, Lu BW, Li SY. 1988. Reproductive behavior of brow-antlered deer, Cervus eldi hainanus [J]. Acta Theriologica Sinica, 8(2): 89-94. [袁喜才，卢柏威，李善元. 1988. 海南坡鹿繁殖习性的研究. 兽类学报, 8(2): 89-94.]

Yuan XC, Lu CH, Yun DX, Mo YN, Fu DL, Liu DG. 1995. Domestication and development of eld's deer fawn [J]. Chinese Wildlife, 16(6): 32-35. [袁喜才, 卢成华, 冯 敏, 云大兴, 莫燕妮, 符大亮, 刘德 光. 1995. 坡鹿幼仔驯养与生长发育. 野生动物, 16(6): 32-35.]

Zhang LC, Li YC. 2007. Review of animal sexual segregation [J]. Journal of Hainan Normal University (Nature Science Edition), 20(3): 272-276. [张利存, 李玉春. 2007. 动物性别分离机制的研究进展. 海南师范大学学报, 20(3): 272-276.]

Zhang Q, Ji YJ, Zeng ZG, Song YL, Zhang DX. 2007. Influence of the founder effect on genetic diversity of translocated populations: An example from Hainan Eld's Deer [J]. Chinese Journal of Zoology, 42(3): 54-60. [张 琼, 吉亚杰，曾治高，宋延龄，张德兴. 2007. 奠基者效应对海南坡鹿迁地保护种群遗传多样性的影响. 动物学 杂志, 42(3): 54-60.] 